TRANSACTIONS OF THE

AMERICAN MATHEMATICAL SOCIETY

Volume 353, Number 6, Pages 2171-2192

S 0002-9947(01)02685-X

Article electronically published on February 7, 2001

\title{
GROUPS WITH TWO EXTREME CHARACTER DEGREES AND THEIR NORMAL SUBGROUPS
}

\author{
GUSTAVO A. FERNÁNDEZ-ALCOBER AND ALEXANDER MORETÓ
}

\begin{abstract}
We study the finite groups $G$ for which the set $\operatorname{cd}(G)$ of irreducible complex character degrees consists of the two most extreme possible values, that is, 1 and $|G: Z(G)|^{1 / 2}$. We are easily reduced to finite $p$-groups, for which we derive the following group theoretical characterization: they are the p-groups such that $|G: Z(G)|$ is a square and whose only normal subgroups are those containing $G^{\prime}$ or contained in $Z(G)$. By analogy, we also deal with $p$ groups such that $|G: Z(G)|=p^{2 n+1}$ is not a square, and we prove that $\operatorname{cd}(G)$ $=\left\{1, p^{n}\right\}$ if and only if a similar property holds: for any $N \unlhd G$, either $G^{\prime} \leq N$ or $|N Z(G): Z(G)| \leq p$. The proof of these results requires a detailed analysis of the structure of the $p$-groups with any of the conditions above on normal subgroups, which is interesting for its own sake. It is especially remarkable that these groups have small nilpotency class and that, if the nilpotency class is greater than 2, then the index of the centre is small, and in some cases we may even bound the order of $G$.
\end{abstract}

\section{INTRODUCTION}

The study of the structure of a finite group $G$ by imposing conditions on the set $\operatorname{cd}(G)$ of the degrees of its complex irreducible characters (henceforth, referred to simply as character degrees) has been considered in many research papers in the last decades. For example, groups having just two different character degrees are solvable, and these groups have been thoroughly investigated: see the results by Isaacs and Passman in [13, 14, Isaacs' book [12, Chapter 12] or Bannuscher's papers [1, 2]. On the other hand, it is well-known that the degree of an irreducible character cannot exceed $|G: Z(G)|^{1 / 2}$. Groups for which this bound is attained are called groups of central type. In 1982 Howlett and Isaacs 9 proved that a group of central type must be solvable, but not necessarily nilpotent.

In this paper, we begin by studying the groups satisfying both conditions above, that is, groups for which $\operatorname{cd}(G)=\left\{1,|G: Z(G)|^{1 / 2}\right\}$. Since $\operatorname{cd}(G / Z(G)) \subseteq \operatorname{cd}(G)$ and the order of $G / Z(G)$ is the sum of the squares of the degrees of its irreducible characters, it follows that $G / Z(G)$ is abelian, and so $G$ is nilpotent of class 2 . But $G$ has only two character degrees; hence all the Sylow subgroups of $G$ but one are abelian, and this one is also of central type with two character degrees. This allows us to reduce ourselves to the case of $p$-groups, and we will do so in the sequel. Thus our problem can also be related to the investigation of the non-linear irreducible

Received by the editors June 9, 1999 and, in revised form, December 8, 1999.

2000 Mathematics Subject Classification. Primary 20C15, $20 \mathrm{D} 15$.

Research of the second author supported by a grant of the Basque Government and by the University of the Basque Country grant UPV 127.310-EB160/98. 
characters of minimum degree (also called minimal characters) in a finite $p$-group, initiated by Mann in his recent paper [17]: we deal with the groups whose minimal characters have maximum possible degree.

Some partial results are known about the $p$-groups under consideration. For example, the case when all the non-linear characters are faithful is treated in 5 . Lemma 1]: these $p$-groups are characterized by the conditions that $Z(G)$ is cyclic and $\left|G^{\prime}\right|=p$ (the result is due to Huppert). Also, Theorem 7.5 in Huppert's book [1] shows that $\operatorname{cd}(G)=\left\{1,|G: Z(G)|^{1 / 2}\right\}$ for any finite group of class 2 such that $[x, G]=G^{\prime}$ for all non-central elements $x$. In [18, Lemma 5.4] Noritzsch proves that the $p$-groups of central type with two character degrees such that $Z(G)=G^{\prime}$ are precisely the semiextraspecial $p$-groups, that is, $p$-groups for which the factor group over any maximal subgroup of the centre is extraspecial. These groups are also the Camina $p$-groups of class 2. (Following [4, we say that $G$ is a Camina group when $\{[x, g]: g \in G\}=G^{\prime}$ for any $x \in G \backslash G^{\prime}$. Camina groups have been widely studied in the literature: see 4 and the references there.)

Our first theorem, of an elementary nature, aims at extending some of the results above to arbitrary $p$-groups of central type with two character degrees, thus obtaining several characterizations of these groups.

Theorem A. For a non-abelian p-group $G$, the following conditions are equivalent:

(i) $\operatorname{cd}(G)=\left\{1,|G: Z(G)|^{1 / 2}\right\}$.

(ii) $\mathrm{Cl}_{G}(x)=x G^{\prime}$ for all $x \in G \backslash Z(G)$, that is, the set of conjugacy class lengths of $G$ is $\left\{1,\left|G^{\prime}\right|\right\}$.

(iii) $G^{\prime}=[x, G]$ for all $x \in G \backslash Z(G)$.

(iv) $G$ is isoclinic to a semiextraspecial p-group, i.e. to a Camina p-group of class 2.

(v) $Z(G / N)=Z(G) / N$ for any normal subgroup $N$ of $G$ such that $G^{\prime} \not \leq N$.

We note that the equivalence between (i) and (ii) can also be deduced from Bannuscher's paper [1, Part I] by combining his theorems 1.2 and 2.8, where he characterizes the nilpotent groups $G$ with the following property: if $\chi, \psi$ are distinct non-linear irreducible characters of $G$ and $\chi_{Z(G)}=\chi(1) \lambda, \psi_{Z(G)}=\psi(1) \mu$ with $\lambda, \mu$ linear characters of $Z(G)$, then $\lambda \neq \mu$. Also, this equivalence is another example of the "duality" between results about character degrees and conjugacy class lengths: groups with two extreme character degrees are exactly the groups with two extreme class lengths. On the other hand, Verardi [20, Theorem 1.2] proves that (i) and (ii) are equivalent under the additional assumption that $G^{\prime}=Z(G)$; that is, he gives a characterization of semiextraspecial groups.

Our next goal is to show that we may characterize the $p$-groups $G$ such that $\operatorname{cd}(G)=\left\{1,|G: Z(G)|^{1 / 2}\right\}$ by the position of their normal subgroups. Observe that, for any $N \unlhd G$, either $G^{\prime} \leq N$ or $\operatorname{cd}(G / N)=\left\{1,|G: Z(G)|^{1 / 2}\right\}$. In the latter case

$$
|G: Z(G)| \leq|G / N: Z(G / N)| \leq|G / N: Z(G) N / N|=|G: Z(G) N|,
$$

whence $N \leq Z(G)$. So in some sense these groups have no "non-trivial" normal subgroups. The following result shows that the converse also holds, under the natural condition that $|G: Z(G)|$ is a square.

Theorem B. Let $G$ be a p-group such that $|G: Z(G)|=p^{2 n}$ is a square. Then the following statements are equivalent: 
(i) $\operatorname{cd}(G)=\left\{1, p^{n}\right\}$.

(ii) The normal subgroups of $G$ either contain $G^{\prime}$ or are contained in $Z(G)$.

We have mentioned before that there was no need of dealing with finite nilpotent groups in general and that we could reduce ourselves to $p$-groups. If we want to trace back the information in Theorems $\mathrm{A}$ and $\mathrm{B}$ in order to get results about arbitrary finite nilpotent groups, it turns out that these theorems continue to hold after the obvious modifications in the statements where the prime $p$ is involved.

Within the context of $p$-groups it seems natural to consider also the case when $|G: Z(G)|=p^{2 n+1}$ is not a square and $\operatorname{cd}(G)=\left\{1, p^{n}\right\}$. These groups are easily seen to have class at most 3 , and Theorem B suggests trying to characterize them in terms of their normal subgroups, which we do in the following theorem.

Theorem C. Let $G$ be a p-group such that $|G: Z(G)|=p^{2 n+1}$ is not a square. Then the following statements are equivalent:

(i) $\operatorname{cd}(G)=\left\{1, p^{n}\right\}$.

(ii) For any $N \unlhd G$, either $G^{\prime} \leq N$ or $|N Z(G): Z(G)| \leq p$.

However, the duality between character degrees and conjugacy class lengths that was observed after Theorem A does not hold for the p-groups in Theorem C: for instance, any $p$-group of maximal class of order $p^{4}$ has character degrees 1 and $p$, but the conjugacy class lengths are $1, p$ and $p^{2}$.

The previous theorems lead us to the following definition.

Definition. We say that a $p$-group $G$ satisfies the strong condition on normal subgroups provided that, for any $N \unlhd G$, either $G^{\prime} \leq N$ or $N \leq Z(G)$. Similarly, $G$ satisfies the weak condition on normal subgroups when, for any $N \unlhd G$, either $G^{\prime} \leq N$ or $|N Z(G): Z(G)| \leq p$.

Thus Theorems B and C concern p-groups with the strong or weak condition on normal subgroups, respectively. The difficulty in the proof of these theorems lies in showing that (ii) implies (i), which is performed by means of a thorough analysis of the structure of the groups in question. We summarize the information we have obtained about these groups in the next theorems.

Theorem D. Let $G$ be a p-group of class 2 .

(i) If $G$ satisfies the strong condition on normal subgroups, then $\exp G / Z(G)=$ $\exp G^{\prime}=p$.

(ii) If $G$ satisfies the weak condition on normal subgroups, then $\exp G / Z(G)=$ $\exp G^{\prime}=p$ or $p^{2}$. Moreover, in the latter case $G / Z(G) \cong C_{p^{2}} \times C_{p^{2}}$ and $G^{\prime} \cong C_{p^{2}}$.

Theorem E. Let $G$ be a p-group of class 3 satisfying the weak condition on normal subgroups. Then $\left|Z_{2}(G): Z(G)\right| \leq p^{2}$, and if $|G: Z(G)|$ is not a square then $\left|Z_{2}(G): Z(G)\right|=p$.

The following theorem shows that the $p$-groups with either the strong or weak condition on normal subgroups have small nilpotency class and, when it is greater than 2, the index of the centre is small. In other words, we could say that "most" of these groups have class 2. Of course, as extraspecial groups show, the index of the centre cannot be bounded when the class is 2 .

Theorem F. Let $G$ be a p-group. 
(i) If $G$ satisfies the strong condition on normal subgroups, then it has nilpotency class $c \leq 3$, and if $c=3$ then $|G: Z(G)|=p^{3}$.

(ii) If $G$ satisfies the weak condition on normal subgroups, then it has nilpotency class $c \leq 4$. If $c=4$ then $|G: Z(G)|=p^{4}$, whereas for $c=3$ we have $|G: Z(G)|=p^{3}, p^{4}$ or $p^{6}$ for odd $p$ and $|G: Z(G)|=2^{3}$ or $2^{4}$ when $p=2$.

We will provide examples showing that all the distinct possibilities for $|G: Z(G)|$ listed in Theorem F actually occur. Surprisingly, for odd $p$ there is a gap at $p^{5}$ among the values that the index of the centre can take in a $p$-group with the weak condition on normal subgroups and class greater than 2. Observe also the different behaviour of the prime 2 in this same case.

Now Theorems B and C follow easily from these results. As we have already pointed out in the abstract, we think that the $p$-groups with either of the conditions on normal subgroups introduced above deserve study for their own sake; and, for this reason, we consider Theorems D, E and F among the main theorems in this paper. Also, we have developed our study of these groups a little further than necessary for our initial purpose and have obtained the following important theorem, which shows that in many of the cases in Theorem F it is not only the index of the centre of $G$ that is small, but even the order of $G$.

Theorem G. Let $G$ be a p-group.

(i) If $G$ satisfies the strong condition on normal subgroups and has nilpotency class 3, then $|G| \leq p^{5}$. Furthermore, if $p=2$ then $|G|=2^{4}$, that is, $G$ has maximal class.

(ii) If $G$ satisfies the weak condition on normal subgroups and has nilpotency class 4 , then $|G| \leq p^{6}$. Furthermore, if $p=2$ then $|G|=2^{5}$, that is, $G$ has maximal class.

(iii) If $G$ satisfies the weak condition on normal subgroups, has nilpotency class 3 and $|G: Z(G)|=p^{6}$, then $G$ has bounded order. In fact, $|G| \leq p^{18}$.

Since the $p$-groups of order at most $p^{6}$ are completely determined up to isomorphism (see 6, 15), it would be routine to classify the groups in either (i) or (ii) above. On the other hand, we will give examples showing that the order of $G$ cannot be bounded if $G$ has the weak condition on normal subgroups, class 3 and $|G: Z(G)|=p^{3}$ or $p^{4}$.

We want to emphasize that the proofs of Theorems D to $G$ that we present in this paper are purely group theoretical, even though some parts could also be proved by using characters.

Now we explain the distribution of the results in the paper. First of all, in Section 2 we collect some well-known facts which will be frequently used in the rest of the article. After that we prove Theorem A in Section 3. We begin to study the structure of the groups with the strong or weak condition on normal subgroups in Section 4, where besides some elementary results we obtain Theorem D. Then we devote Section 5 to tackling the hardest case, namely that of the groups with the weak condition and class 3, and prove Theorem E. Once this is accomplished, it is not particularly difficult to derive Theorems F and G in Sections 6 and 7, respectively, except for the different behaviour of the prime 2 in Theorem F, which requires careful reasoning. Finally, in Section 8 we come back to our original character theoretical problem and end the paper by proving Theorems B and $\mathrm{C}$. 
We close this introduction with some words about the notation we will use throughout the paper. All groups considered will be finite. For any group $G$, $d(G)$ denotes the minimal number of generators of $G$. On the other hand, $k(G)$ will stand for the number of conjugacy classes of $G$, and $\operatorname{Irr}(G)$ for the set of its irreducible characters. If $G$ is nilpotent, we write $c(G)$ for its nilpotency class. Also, $Z_{i}(G)$ denotes the $i$-th center of $G$ and $\gamma_{i}(G)=\left[G, .{ }_{i}, G\right]$ is the $i$-th term of the lower central series of $G$. If $x \in G$, we define $[x, G]$ as the subgroup generated by all commutators $[x, g]$ with $g \in G$. Finally, if $G$ is a $p$-group, then $\Omega_{i}(G)$ denotes the subgroup generated by the elements of order $\leq p^{i}$, and $\mho_{i}(G)=\left\langle x^{p^{i}} \mid x \in G\right\rangle$.

Acknowledgements. We are very much indebted to Professors I. Martin Isaacs and Avinoam Mann for their helpful suggestions, which have helped us improve the results in this paper. We would also like to thank Professor Isaacs for his remarks about the organization of the paper, which have made us rewrite it in a, we hope, clearer way.

\section{Preliminary Lemmas}

The aim of this section is to collect several well-known facts that will be widely applied in the rest of the paper. We begin by recalling some commutator identities.

Lemma 2.1. Let $G$ be a group and $x, y, z \in G$.

(i) If one of the elements $x, y, z$ belongs to $Z_{2}(G)$, then $[x y, z]=[x, z][y, z]$.

(ii) If $x$ or $y$ lies in $Z_{2}(G)$, then $\left[x^{n}, y\right]=[x, y]^{n}$ for any $n \in \mathbb{Z}$.

(iii) If $x \in Z_{2}(G)$, then $[x, G]=\{[x, g] \mid g \in G\}$.

In particular, all these conclusions hold when $G$ is a group of class 2 .

The following lemma is an immediate consequence of the previous one.

Lemma 2.2. Let $G$ be a p-group and $H \leq Z_{2}(G)$. Then we have that $\left[\mho_{n}(H), G\right]=$ $\mho_{n}([H, G])=\left[H, \mho_{n}(G)\right]$ for any $n \in \mathbb{N}$, and, as a consequence, $\exp [H, G]=$ $\exp H Z(G) / Z(G)$. In particular, if $G$ has class 2 , then $\exp G^{\prime}=\exp G / Z(G)$.

The following commutator identity will be important in Section 5, where we deal with groups of class 3 .

Lemma 2.3. If $G$ is a nilpotent group of class $\leq 3$, then

$$
\left[x^{n}, y\right]=[x, y]^{n}[x, y, x]^{n(n-1) / 2}
$$

for any $x, y \in G$ and $n \in \mathbb{Z}$.

The next lemma is simply a consequence of the dimension of any symplectic space being even.

Lemma 2.4. If $G$ is a p-group such that $\left|G^{\prime}\right|=p$, then $|G: Z(G)|$ is a square.

We have not been able to find any reference to the following result in the literature, although it seems to be well-known. The proof we present here is due to I.M. Isaacs and A. Mann.

Lemma 2.5. Let $P$ be a p-group such that $\left|P^{\prime}\right|=p$ and $|P: Z(P)|>p^{2}$. Then $P$ is not capable; that is, $P$ cannot be written as $G / Z(G)$ for any group $G$. 
Proof. Suppose by way of contradiction that $P=G / Z(G)$. Since $\left|P^{\prime}\right|=p$, we can view $P / Z(P)$ as a symplectic space over $\mathbb{F}_{p}$, where the symplectic form is induced from commutation in $P$. By considering a symplectic basis in $P / Z(P)$ and lifting its elements back to $P$, we can decompose $P$ as a central product of two nonabelian subgroups (take into account here that $|P: Z(P)|>p^{2}$ ). We can thus write $G=X Y$, where $[X, Y] \leq Z(G)$ and $X^{\prime} Z(G)=Y^{\prime} Z(G)=G^{\prime} Z(G)$. Since $[X, Y, Y]=1$, P. Hall's three subgroup lemma yields that $\left[Y^{\prime}, X\right]=1$, and it follows that $\left[G^{\prime}, X\right]=1$. We have similarly that $\left[G^{\prime}, Y\right]=1$. Consequently $G^{\prime} \leq Z(G)$, which is clearly a contradiction.

\section{Proof of Theorem A}

In this section we prove Theorem $\mathrm{A}$, which gives several elementary characterizations of the $p$-groups $G$ such that $\operatorname{cd}(G)=\left\{1,|G: Z(G)|^{1 / 2}\right\}$. This theorem appears as Theorem 3.1 below. The characterization provided by part (iv) of the theorem in terms of isoclinism is due to A. Mann. Before proceeding to the proof of the theorem, let us recall the concept of isoclinic groups. This idea, which generalizes that of isomorphic groups, was introduced by P. Hall in the second of his famous papers on $p$-groups [7]. It is of great importance in the classification of $p$-groups of low order: instead of classifying them directly, one first arranges them in families of isoclinic groups.

Definition. Let $G$ and $H$ be two groups. We say that $G$ and $H$ are isoclinic if there exist isomorphisms $\alpha: G / Z(G) \rightarrow H / Z(H)$ and $\beta: G^{\prime} \rightarrow H^{\prime}$ such that the diagram

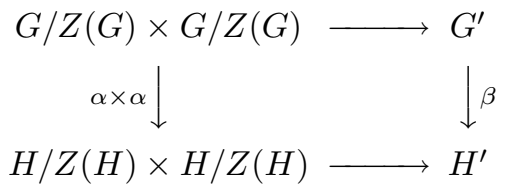

is commutative, where the horizontal arrows denote the maps induced from commutation in $G$ and $H$, respectively.

Among the results that Hall proves in [7, we point out the following two:

(i) Two isoclinic nilpotent groups have the same nilpotency class.

(ii) For any group $G$ there exists at least one group $H$ isoclinic to $G$ such that $Z(H) \leq H^{\prime}$. Furthermore, if $G$ is a $p$-group, so is $H$.

By combining these properties we deduce in particular that any $p$-group of class 2 is isoclinic to a $p$-group $H$ such that $Z(H)=H^{\prime}$.

Theorem 3.1. For a non-abelian p-group $G$, the following conditions are equivalent:

(i) $\operatorname{cd}(G)=\left\{1,|G: Z(G)|^{1 / 2}\right\}$.

(ii) $\mathrm{Cl}_{G}(x)=x G^{\prime}$ for all $x \in G \backslash Z(G)$; that is, the set of conjugacy class lengths of $G$ is $\left\{1,\left|G^{\prime}\right|\right\}$.

(iii) $G^{\prime}=[x, G]$ for all $x \in G \backslash Z(G)$.

(iv) $G$ is isoclinic to a semiextraspecial p-group, i.e. to a Camina p-group of class 2.

(v) $Z(G / N)=Z(G) / N$ for any normal subgroup $N$ of $G$ such that $G^{\prime} \not \leq N$. 
Proof. We start by proving that (i) implies (ii). Let $t$ be the number of nonlinear irreducible characters of $G$. Since $\operatorname{cd}(G)=\left\{1,|G: Z(G)|^{1 / 2}\right\}$, we have that $|G|=\left|G / G^{\prime}\right|+t|G / Z(G)|$, that is, $t=|Z(G)|-\left|Z(G) / G^{\prime}\right|$. It follows that $k(G)=|\operatorname{Irr}(G)|=\left|G / G^{\prime}\right|+|Z(G)|-\left|Z(G) / G^{\prime}\right|$ and $G$ has $\left|G / G^{\prime}\right|-\left|Z(G) / G^{\prime}\right|$ noncentral classes. As each of these classes has at most $\left|G^{\prime}\right|$ elements and the union of them is $G \backslash Z(G)$, we necessarily have that $\mathrm{Cl}_{G}(x)=x G^{\prime}$ for any non-central $x$.

It is clear that (iii) follows from (ii), and (iii) implies (i) according to the result in Huppert's book mentioned in the introduction. (Just note that a non-abelian nilpotent group $G$ such that $[x, G]=G^{\prime}$ for all $x \in G \backslash Z(G)$ necessarily has class 2: if $g \in G^{\prime}$ then $[g, G] \leq\left[G^{\prime}, G\right]<G^{\prime}$ and so $g \in Z(G)$.)

Let us see now that (iii) and (iv) are equivalent. For this purpose, observe that the property that $[x, G]=G^{\prime}$ for all $x \in G \backslash Z(G)$ is preserved when passing to an isoclinic group: this follows easily from the commutativity of the diagram (3). Thus $G$ satisfies (iii) if and only if some group isoclinic to $G$ does. This already proves that (iv) implies (iii) by the very definition of Camina groups (note that $H^{\prime}=Z(H)$ in any Camina group of class 2). On the other hand, if (iii) holds then $G$ has class 2 and, as argued just before the statement of the theorem, $G$ is isoclinic to a $p$-group $H$ such that $H^{\prime}=Z(H)$. Then $H$ also satisfies property (iii) and is consequently a Camina group of class 2 .

Finally, we prove that (i) and (v) are equivalent. Suppose (i) holds, and let $N$ be a normal subgroup of $G$. As already said in the introduction, if $G^{\prime} \not \leq N$ then $N \leq Z(G)$ and it follows from formula (11) that $Z(G / N)=Z(G) / N$. Conversely, suppose (v) holds. Observe that this implies that $c(G)=2$. Let $\chi$ be a non-linear irreducible character of $G$. Then $G^{\prime} \not \leq \operatorname{Ker} \chi$ and consequently $Z(\chi) / \operatorname{Ker} \chi=$ $Z(G / \operatorname{Ker} \chi)=Z(G) / \operatorname{Ker} \chi$, that is, $Z(\chi)=Z(G)$. Since $G / Z(\chi)$ is abelian, we conclude from [12, Theorem 2.31] that $\chi(1)^{2}=|G: Z(\chi)|=|G: Z(G)|$.

\section{Proof of Theorem D}

In this section we begin to study the $p$-groups with either the strong or the weak condition on normal subgroups. First of all, observe that both conditions are obviously hereditary for quotients. This is a fact that we will use freely in the sequel. However, the behaviour of these conditions with respect to direct products is not so smooth. In fact, if $P$ and $Q$ are non-abelian $p$-groups that satisfy the same condition on normal subgroups, then $P \times Q$ does not, since either of the factors is a normal subgroup which does not fulfill the required property. Even the direct product by a non-trivial abelian $p$-group $A$ does not preserve these conditions. For example, a $p$-group of maximal class $P$ of order $p^{4}$ satisfies the strong condition, but $P \times A$ never does: choose elements $x \in P^{\prime} \backslash \gamma_{3}(P)$ and $1 \neq a \in A$ and consider the subgroup $N=\langle x a\rangle \gamma_{3}(P)$ of $P \times A$. In the case of the weak condition we may reason similarly with a $p$-group of maximal class of order $p^{5}$. In particular, these conditions are not preserved under isoclinism. However, as a consequence of Theorems $\mathrm{B}$ and $\mathrm{C}$ we will be able to prove that there are many cases in which these conditions are preserved by forming a direct product with an abelian $p$-group.

The class of a $p$-group with the strong or weak condition on normal subgroups is very small, as we see in our next result. Observe that this statement is included in Theorem $\mathrm{F}$ in the introduction.

Proposition 4.1. Let $G$ be a p-group. If $G$ satisfies the strong condition on normal subgroups, then $c(G) \leq 3$, and if $G$ satisfies the weak condition, then $c(G) \leq 4$. 
Proof. Let us assume first that $G$ satisfies the strong condition on normal subgroups. If $c(G) \geq 2$ then $\left|Z_{2}(G): Z(G)\right| \geq p$, and it follows that $G^{\prime} \leq Z_{2}(G)$. Hence $\gamma_{4}(G)=\left[G^{\prime}, G, G\right]=1$ and $c(G) \leq 3$. The proof when $G$ satisfies the weak condition follows from the same argument, by observing that if $c(G) \geq 3$ then $\left|Z_{3}(G): Z(G)\right| \geq p^{2}$.

These bounds for the nilpotency class are sharp, as is shown by the $p$-groups of maximal class and order $p^{4}$ and $p^{5}$, respectively.

Since the class of the $p$-groups with either of the conditions on normal subgroups is very small, it makes sense to study them in separate cases by fixing the class. In the next theorem, which corresponds to Theorem $\mathrm{D}$ in the introduction, we consider the case when the class is 2 and see that, although it is not possible to bound the index of the centre in these groups, we may control the exponent of $G / Z(G)$, which is $p$ or $p^{2}$. The proof of this fact presented here is a simplification, due to I.M. Isaacs, of a previous proof by the authors. We also owe him the idea of studying the structure of $G / Z(G)$ when $\exp G / Z(G)=p^{2}$, a problem which is also solved in Theorem D.

Before the theorem we need a very simple result, which we state as a lemma since it will be also used in two other proofs in this paper.

Lemma 4.2. Let $G$ be a p-group and $M$ a maximal subgroup of $G^{\prime}$ which is normal in $G$. Choose a normal subgroup $N$ of $G$ which is maximal with respect to the condition $N \cap G^{\prime}=M$, and let $K / N=Z(G / N)$. Then the following properties hold:

(i) $G / K$ is elementary abelian and has square order.

(ii) $K / N$ is cyclic.

Proof. Since the derived subgroup of $G / N$ has order $p$, it follows from Lemma 2.2 that $G / K$ is elementary abelian, and by Lemma 2.4 it has square order. On the other hand, suppose $K / N$ is not cyclic. Then $K / N$ has more than one subgroup of order $p$, one of which is $G^{\prime} N / N$. If we choose a different one, say $L / N$, then $L \unlhd G$ and $L \cap G^{\prime}=M$. This contradicts the choice of $N$.

Theorem 4.3. Let $G$ be a p-group of class 2 .

(i) If $G$ satisfies the strong condition on normal subgroups, then $\exp G / Z(G)=$ $\exp G^{\prime}=p$.

(ii) If $G$ satisfies the weak condition on normal subgroups, then $\exp G / Z(G)=$ $\exp G^{\prime}=p$ or $p^{2}$. Moreover, in the latter case $G / Z(G) \cong C_{p^{2}} \times C_{p^{2}}$ and $G^{\prime} \cong C_{p^{2}}$.

Proof. Let $e$ be the common exponent of $G / Z(G)$ and $G^{\prime}$. If we decompose the abelian group $G / Z(G)$ as a direct product of cyclic subgroups, $G / Z(G)=\left\langle\bar{x}_{1}\right\rangle \times$ $\cdots \times\left\langle\bar{x}_{k}\right\rangle$, then we have that $G^{\prime}=\left\langle\left[x_{i}, x_{j}\right] \mid i, j=1, \ldots, k\right\rangle$. The following observation will be essential in the proof: $G / Z(G)$ has at least two cyclic factors of order $e$. (Just note that if we choose a commutator $\left[x_{i}, x_{j}\right]$ of order $e$, then both $\bar{x}_{i}$ and $\bar{x}_{j}$ have order $e$ in $G / Z(G)$.)

Let $M$ be any maximal subgroup of $G^{\prime}$. Since $G$ has class $2, M$ is normal in $G$ and we may apply Lemma4.2. Thus if we choose $N$ normal in $G$ and maximal with the property that $G^{\prime} \cap N=M$ and let $K / N=Z(G / N)$, then $G / K$ is elementary abelian and $K / N$ is cyclic. 
Suppose now that $G$ satisfies the strong condition on normal subgroups. Then $N \leq Z(G)$ and consequently $K / Z(G)$ is cyclic. Since $G / K$ is elementary abelian, $G / Z(G)$ cannot have two cyclic factors of order greater than $p$. Hence $e=p$, and we have proved (i).

If $G$ satisfies the weak condition, then $|N Z(G): Z(G)| \leq p$, and we derive that $K / Z(G)$ has rank at most 2 and that it has at most one cyclic factor of order exceeding $p$. By taking into account again that $G / K$ is elementary and the abovementioned observation that $G / Z(G)$ has at least two cyclic factors of order $e$, we deduce that either $e=p$, or else $e=p^{2}$ and $G / Z(G)$ has exactly two cyclic factors of order $p^{2}$. In the latter case, $G^{\prime}$ has just one factor of order $p^{2}$, and thus we may choose $M=\Omega_{1}\left(G^{\prime}\right)$. Let $T / Z(G)=\Omega_{1}(G / Z(G))$. Then Lemma 2.2 yields that $\exp [T, G]=\exp T / Z(G)=p$, and consequently $[T, G] \leq M$. Hence $T \leq K$ and, since $K / Z(G)$ has rank at most 2 , we obtain that $\left|\Omega_{1}(G / Z(G))\right| \leq p^{2}$. It follows that $G / Z(G) \cong C_{p^{2}} \times C_{p^{2}}$ and $G^{\prime} \cong C_{p^{2}}$.

Observe that the group $H=\left\langle a, b \mid a^{p^{4}}=b^{p^{2}}=1, a^{b}=a^{1+p^{2}}\right\rangle$ yields an example of a $p$-group of class 2 with the weak condition on normal subgroups such that $\exp H / Z(H)=\exp H^{\prime}=p^{2}$. Furthermore, it is clear that any $p$-group $G$ of class 2 such that $G / Z(G) \cong C_{p^{2}} \times C_{p^{2}}$ and $G^{\prime} \cong C_{p^{2}}$ is isoclinic to $H$. In particular, all the $p$-groups of class 2 with the weak condition on normal subgroups and $\exp G / Z(G)=\exp G^{\prime}=p^{2}$ are isoclinic to $H$. Nevertheless, not all groups isoclinic to $H$ satisfy the weak condition: consider the group $\langle a, b, c| a^{p^{2}}=b^{p^{2}}=$ $\left.c^{p^{2}}=1,[a, b]=c,[a, c]=[b, c]=1\right\rangle$ and note that $\left\langle a^{p}, b^{p}, c^{p}\right\rangle$ is a normal subgroup.

\section{Proof of Theorem E}

The proof of Theorem $\mathrm{E}$ is quite elaborate, and for this reason we divide it into Theorems 5.2 and 5.3 below. First we need the following lemma.

Lemma 5.1. Let $G$ be a p-group that satisfies the weak condition on normal subgroups, and define $L$ by means of $L / Z(G)=\Omega_{1}\left(Z_{2}(G) / Z(G)\right.$ ). Then the following holds:

(i) If $[L, G] \leq K \unlhd G$, then $G / K$ has the strong condition on normal subgroups. In particular, $G / Z(G)$ satisfies the strong condition.

Moreover, if $G$ has class 3 then:

(ii) The groups $G / Z_{2}(G), G^{\prime} Z(G) / Z(G)$ and $G^{\prime} /\left(G^{\prime} \cap Z(G)\right)$ have exponent $p$.

(iii) $\gamma_{3}(G) \leq[L, G]$, and these two groups have exponent $p$.

(iv) $G^{\prime}$ and $Z_{2}(G) / Z(G)$ have exponent at most $p^{2}$.

Proof. (i) Suppose $N / K \unlhd G / K$ and $(G / K)^{\prime} \not \leq N / K$. Since $G$ has the weak condition on normal subgroups, we deduce that $|N Z(G): Z(G)| \leq p$ and $N Z(G) / Z(G) \leq$ $L / Z(G)$. Then $[N, G] \leq[L, G] \leq K$ and $N / K \leq Z(G / K)$, as desired.

(ii) It suffices to apply part (i) of Theorem D to $G / Z(G)$.

(iii) We have from part (ii) that $G^{\prime} Z(G) \leq L$, and consequently $\gamma_{3}(G) \leq[L, G]$. On the other hand, $\exp [L, G]=\exp L / Z(G)=p$ by Lemma 2.2.

(iv) It follows from (i) and (iii) that $G /[L, G]$ is a group of class 2 with the strong condition on normal subgroups. Then $\exp G^{\prime} /[L, G]=p$ by Theorem D. Since also $\exp [L, G]=p$, we deduce that $\exp G^{\prime} \leq p^{2}$. Therefore $\exp Z_{2}(G) / Z(G)=$ $\exp \left[Z_{2}(G), G\right] \leq p^{2}$. 
In the remainder, $L$ will denote the subgroup introduced in the previous lemma, and the results there will be used sometimes without further reference.

Theorem 5.2. Let $G$ be a p-group of class 3 that satisfies the weak condition on normal subgroups. Then $Z_{2}(G) / Z(G) \cong C_{p}$ or $C_{p} \times C_{p}$. In particular, $L=Z_{2}(G)$.

Proof. Let us see, first of all, that $Z_{2}(G) / Z(G)$ can only be cyclic when it has order $p$. Indeed, if $Z_{2}(G) / Z(G)=\langle\bar{a}\rangle$ then $Z_{2}(G)=\langle a, Z(G)\rangle$ is abelian. According to Lemma 5.1, $x^{p} \in Z_{2}(G)$ for any $x \in G$, and consequently Lemma 2.1]yields that $1=\left[x^{p}, a\right]=[x, a]^{p}=\left[x, a^{p}\right]$. It follows that $a^{p} \in Z(G)$ and $\left|Z_{2}(G) / Z(G)\right|=p$.

If $\left|Z_{2}(G) / Z(G)\right| \leq p^{2}$, then it follows that $Z_{2}(G) / Z(G) \cong C_{p}$ or $C_{p} \times C_{p}$. Suppose now that $\left|Z_{2}(G): Z(G)\right|>p^{2}$. For any subgroup $N$ such that $Z(G) \leq N \leq Z_{2}(G)$ and $|N: Z(G)|=p^{2}$, we have that $G^{\prime} \leq N$. In other words, any subgroup of $Z_{2}(G) / Z(G)$ of order $p^{2}$ contains $G^{\prime} Z(G) / Z(G)$. Since $Z_{2}(G) / Z(G)$ is not cyclic, it has more than one subgroup of order $p^{2}$ and they all intersect in $G^{\prime} Z(G) / Z(G)$, which must have order $p$. Since by Lemma $5.1 \exp Z_{2}(G) / Z(G) \leq p^{2}$, it necessarily follows that $Z_{2}(G) / Z(G) \cong C_{p^{2}} \times C_{p}$, and $G^{\prime} Z(G) / Z(G)=\mho_{1}\left(Z_{2}(G) / Z(G)\right)$. Now we have to prove that, in fact, this possibility does not hold.

Let $T=\left[Z_{2}(G), G\right]$ and observe that $T$ is abelian and that, according to Lemma 2.2, $\exp T=\exp Z_{2}(G) / Z(G)=p^{2}$. Note also that $\mho_{1}(G) \leq Z_{2}(G)$, by Lemma 5.1 part (ii). Consequently, again using Lemma 2.2 we get that

$$
\mho_{1}(T)=\left[Z_{2}(G), \mho_{1}(G)\right] \leq Z_{2}(G)^{\prime},
$$

and, in particular, $Z_{2}(G)$ is not abelian.

Now we divide the proof into several steps in order to make it clearer.

Step 1. $Z\left(Z_{2}(G)\right)=G^{\prime} Z(G)$.

Since $\left[Z_{2}(G), G^{\prime} Z(G)\right]=1$, we have that $G^{\prime} Z(G) \leq Z\left(Z_{2}(G)\right)$. If the equality does not hold, then $\left|Z_{2}(G): Z\left(Z_{2}(G)\right)\right| \leq p$ and $Z_{2}(G)$ is abelian, a contradiction. Step 2. $\left|T: \Omega_{1}(T)\right|=p$.

If we write $Z_{2}(G)=\left\langle x, y, Z\left(Z_{2}(G)\right)\right\rangle$, then $Z_{2}(G)^{\prime}=\langle[x, y]\rangle$ is cyclic. Since the abelian group $T$ has exponent $p^{2}$, it follows from (4) that $\left|\mho_{1}(T)\right|=p$. Hence $\left|T: \Omega_{1}(T)\right|=p$.

Step 3. If $\bar{G}=G / \Omega_{1}\left(G^{\prime}\right)$, then there exists a maximal subgroup $M$ of $G^{\prime}$ such that $\bar{G}^{\prime}=\bar{T} \times \bar{M}$. Furthermore, $M$ is a normal subgroup of $G$.

Since $G^{\prime}$ is abelian, we have that $\Omega_{1}(T)=T \cap \Omega_{1}\left(G^{\prime}\right)$. Then

$$
|\bar{T}|=\left|T \Omega_{1}\left(G^{\prime}\right): \Omega_{1}\left(G^{\prime}\right)\right|=\left|T: T \cap \Omega_{1}\left(G^{\prime}\right)\right|=\left|T: \Omega_{1}(T)\right|=p,
$$

according to the previous step. Hence we can write $\bar{G}^{\prime}=\bar{T} \times \bar{M}$ with $M \max G^{\prime}$, since $\bar{G}^{\prime}$ is elementary abelian by Lemma 5.1. Finally, we also know from Lemma 5.1 that $\exp \gamma_{3}(G)=p$, and consequently $\bar{G}$ has class 2 . This explains the normality of $M$ in $G$.

Step 4. Let $N$ be normal in $G$ and maximal with respect to the condition that $N \cap G^{\prime}=M$. If $K / N=Z(G / N)$, then $\left|K: G^{\prime} Z(G)\right|=p$.

We know from Lemma 4.2 that $K / N$ is cyclic. Also, $G^{\prime} \not \leq N$ implies that $|N Z(G): Z(G)| \leq p$. Since $G^{\prime} Z(G)=M T Z(G)=M Z(G) \leq N Z(G)$, it follows that $G^{\prime} Z(G)=N Z(G)$. In particular, $K / G^{\prime} Z(G)$ is cyclic.

On the other hand, by the definition of $K$ we have that $[K, G] \leq N \cap G^{\prime}=M$. Consequently $\left[K, Z_{2}(G)\right] \leq M \cap T \leq \Omega_{1}\left(G^{\prime}\right)$ and, by Lemma [2.2] $\left[\mho_{1}(K), Z_{2}(G)\right]=$ $\mho_{1}\left(\left[K, Z_{2}(G)\right]\right)=1$. Since $\mho_{1}(K) \leq Z_{2}(G)$, we derive from Step 1 that $\mho_{1}(K) \leq$ $G^{\prime} Z(G)$. Hence $\exp K / G^{\prime} Z(G)=p$, and we conclude that $\left|K: G^{\prime} Z(G)\right|=p$. 
Step 5. Final contradiction.

We then have that

$$
|G: Z(G)|=|G: K|\left|K: G^{\prime} Z(G)\right|\left|G^{\prime} Z(G): Z(G)\right|=p^{2}|G: K|
$$

is a square, according to Lemma 4.2, Since $\left|Z_{2}(G): Z(G)\right|=p^{3}$, we deduce that $\left|G: Z_{2}(G)\right|$ is not a square. This is a contradiction, since Lemma 2.4 applied to $G / Z(G)$ yields that $\left|G: Z_{2}(G)\right|$ is a square.

Theorem 5.3. Let $G$ be a p-group of class 3 such that $|G: Z(G)|$ is not a square. If $G$ satisfies the weak condition on normal subgroups, then $\left|Z_{2}(G): Z(G)\right|=p$.

Proof. Suppose that $\left|Z_{2}(G): Z(G)\right| \geq p^{2}$. Bearing in mind Theorem5.2, it follows that $Z_{2}(G) / Z(G) \cong C_{p} \times C_{p}$. We will see how this leads to a contradiction under the hypothesis that $|G: Z(G)|$ is not a square.

We write $\bar{G}=G / Z(G)$ throughout the proof. We first observe that $Z_{2}(G)=$ $G^{\prime} Z(G)$, since otherwise $\left|\bar{G}^{\prime}\right|=p$ and $|\bar{G}: Z(\bar{G})|=\left|G: Z_{2}(G)\right|$ is a square, a contradiction. Recall that $\bar{G}$ satisfies the strong condition on normal subgroups, and that $\exp G / Z_{2}(G)=p$ by Lemma 5.1 In particular, $g^{p} \in Z_{2}(G)$ for all $g \in G$. Again we split the proof into several steps.

Step 1. There exist $a, b \in G$ such that $\bar{G}^{\prime}=\left\langle\bar{a}^{p}\right\rangle \times\left\langle\bar{b}^{p}\right\rangle$ and $\langle\bar{a}, \bar{b}\rangle$ is abelian.

Let $\bar{N}$ be a subgroup of $\bar{G}^{\prime}$ of order $p$, and put $Z(\bar{G} / \bar{N})=\bar{H} / \bar{N}$. Then the derived subgroup of $\bar{G} / \bar{N}$ has order $p$ and, by Lemma $2.4|\bar{G}: \bar{H}|$ is a square. Since $Z(\bar{G}) \leq \bar{H}$ and $|\bar{G}: Z(\bar{G})|$ is not a square, there exists an element $\bar{a} \in \bar{H} \backslash Z(\bar{G})$. Therefore $[\bar{a}, \bar{G}]=\bar{N}$, and $\langle\bar{a}\rangle \bar{N}$ is a normal subgroup of $\bar{G}$ not contained in the centre. It follows that $\bar{G}^{\prime} \leq\langle\bar{a}\rangle \bar{N}$ and, consequently,

$$
\bar{G}^{\prime}=\bar{G}^{\prime} \cap\langle\bar{a}\rangle \bar{N}=\left(\bar{G}^{\prime} \cap\langle\bar{a}\rangle\right) \bar{N}=\left\langle\bar{a}^{p}\right\rangle \bar{N}=\left\langle\bar{a}^{p}\right\rangle \times \bar{N},
$$

by Dedekind's Law and taking into account that $\bar{a}^{p} \in Z(\bar{G})=\bar{G}^{\prime}$. Now we can argue analogously with $\left\langle\bar{a}^{p}\right\rangle$ in place of $\bar{N}$ to obtain that

$$
\bar{G}^{\prime}=\left\langle\bar{a}^{p}\right\rangle \times\left\langle\bar{b}^{p}\right\rangle
$$

for some $\bar{b}$ such that $[\bar{b}, \bar{G}]=\left\langle\bar{a}^{p}\right\rangle$. Since $[\bar{a}, \bar{b}] \in[\bar{a}, \bar{G}] \cap[\bar{b}, \bar{G}] \leq \bar{N} \cap\left\langle\bar{a}^{p}\right\rangle=\overline{1}$, we also deduce that the subgroup $\langle\bar{a}, \bar{b}\rangle$ is abelian.

Step 2. $d\left(G^{\prime}\right) \leq 2$.

If we see that $K=\left\langle a^{p}, b^{p}\right\rangle \mho_{1}\left(G^{\prime}\right)$ is a normal subgroup of $G$, then the weak condition on normal subgroups yields that $G^{\prime} \leq\left\langle a^{p}, b^{p}\right\rangle$, since $\mho_{1}\left(G^{\prime}\right) \leq \Phi\left(G^{\prime}\right)$. By observing that $\left\langle a^{p}, b^{p}\right\rangle \leq G^{\prime} Z(G)$ is abelian, we conclude that $d\left(G^{\prime}\right) \leq 2$.

Thus it is enough to prove that $\left[a^{p}, G\right],\left[b^{p}, G\right] \leq \mho_{1}\left(G^{\prime}\right)$. According to formula (22), it suffices to check that $\left[G^{\prime}, a\right],\left[G^{\prime}, b\right] \leq \mho_{1}\left(G^{\prime}\right)$. Since $G^{\prime} \leq\left\langle a^{p}, b^{p}\right\rangle Z(G)$, we only need to consider the commutators $\left[b^{p}, a\right]$ and $\left[a^{p}, b\right]$. Now it follows from the previous step that the subgroup $\langle a, b\rangle$ has class $\leq 2$ and, consequently, both

$$
\left[a^{p}, b\right]=[a, b]^{p} \quad \text { and } \quad\left[b^{p}, a\right]=[b, a]^{p}
$$

lie in $\mho_{1}\left(G^{\prime}\right)$.

Step 3. $\exp \left(G^{\prime} \cap Z(G)\right)=p$.

According to Lemma 5.1 it is enough to see that $G^{\prime} \cap Z(G)=\gamma_{3}(G)$. We have that $L=G^{\prime} Z(G)$, and therefore $G / \gamma_{3}(G)$ satisfies the strong condition on normal 
subgroups. It follows from Theorem $\mathrm{D}$ that $\exp G^{\prime} / \gamma_{3}(G)=p$, and we deduce from Step 2 that $\left|G^{\prime} / \gamma_{3}(G)\right| \leq p^{2}$. On the other hand,

$$
\left|G^{\prime}: G^{\prime} \cap Z(G)\right|=\left|G^{\prime} Z(G): Z(G)\right|=p^{2} .
$$

Thus the desired equality follows by observing that $\gamma_{3}(G) \leq G^{\prime} \cap Z(G)$.

Step 4. $Z_{2}(G)$ centralizes both $a$ and $b$.

Since $[a, b] \in G^{\prime} \cap Z(G)$, by applying the previous step to (5) we deduce that $\left[a^{p}, b\right]=\left[b^{p}, a\right]=1$. Now the assertion follows from the fact that $Z_{2}(G)=$ $\left\langle a^{p}, b^{p}\right\rangle Z(G)$.

Step 5. Contradiction for $p>2$.

Consider any $x \in G$. Then $x^{p} \in Z_{2}(G)$ commutes with $a$, so that Lemma [2.3 yields

$$
1=\left[x^{p}, a\right]=[x, a]^{p}[x, a, x]^{\left(\begin{array}{c}
p \\
2
\end{array}\right)}=[x, a]^{p},
$$

since $p>2$. On the other hand, for any value of $p$ we have that

$$
\left[a^{p}, x\right]=[a, x]^{p}[a, x, a]^{\left(\begin{array}{c}
p \\
2
\end{array}\right)}=[a, x]^{p},
$$

and we derive that $\left[a^{p}, x\right]=1$ for any $x \in G$. Hence $a^{p} \in Z(G)$ and $\bar{a}^{p}=\overline{1}$, which is impossible.

Step 6. Contradiction for $p=2$.

Since the equality in (6) holds in this case, it suffices to show either that $[a, x]^{2}=$ 1 for any $x \in G$, or that $[b, x]^{2}=1$ for any $x \in G$, and then finish the proof as in the previous step. We begin by proving that $C_{\bar{G}}(\bar{a})=C_{\bar{G}}(\bar{b})$. Let us write $C_{\bar{G}}(\bar{a})=M / Z(G)$, where $M$ is a maximal subgroup of $G$, since the commutator subgroup $[\bar{a}, \bar{G}]$ has order 2 . We claim that $M=C_{G}\left(G^{\prime}\right)$. Indeed, if $u \in M$ then $[a, u] \in G^{\prime} \cap Z(G)$, and we derive that $\left[a^{2}, u\right]=[a, u]^{2}[a, u, a]=1$, by Lemma 2.3 and Step 3. On the other hand $[\bar{b}, \bar{u}]=\overline{1}$ or $\bar{a}^{2}$ and, in any case, $[b, u, u]=1$. It follows that $\left[b^{2}, u\right]=[b, u]^{2}=\left[b, u^{2}\right]=1$, since $u^{2} \in Z_{2}(G)$ commutes with $b$ according to Step 4. We have thus shown that $u$ centralizes both $a^{2}$ and $b^{2}$, whence $u \in C_{G}\left(G^{\prime}\right)$. Since $M$ is maximal in $G$ and $C_{G}\left(G^{\prime}\right)<G$, we derive that $M=C_{G}\left(G^{\prime}\right)$. Although the roles of $\bar{a}$ and $\bar{b}$ are not symmetric, we can find for $\bar{b}$ another element $\bar{c}$ such that $\bar{G}^{\prime}=\left\langle\bar{b}^{2}\right\rangle \times\left\langle\bar{c}^{2}\right\rangle$ and $[\bar{c}, \bar{G}]=\left\langle\bar{b}^{2}\right\rangle$. Arguing as above, it follows that $C_{G}\left(G^{\prime}\right)$ coincides with the inverse image of $C_{\bar{G}}(\bar{b})$ in $G$. We conclude that $C_{\bar{G}}(\bar{a})=C_{\bar{G}}(\bar{b})$.

Now, if $x \in M$, we have that $[a, x],[b, x] \in G^{\prime} \cap Z(G)$ and $[a, x]^{2}=[b, x]^{2}=1$. Suppose otherwise that $x \in G \backslash M$. Since $\bar{G}^{\prime}=\left\langle\bar{a}^{2}\right\rangle \times[\bar{a}, \bar{G}]$ and $[\bar{b}, \bar{G}]=\left\langle\bar{a}^{2}\right\rangle$, it follows that $[\bar{b}, \bar{x}]=\bar{a}^{2}$ and $[\bar{a}, \bar{x}]=\bar{b}^{2}$ or $(\overline{a b})^{2}$. In the former case we have that $[\overline{a b}, \bar{x}]=(\overline{a b})^{2}$. Since $\bar{G}=\langle\bar{x}\rangle C_{\bar{G}}(\overline{a b})$, we deduce that $\langle\overline{a b}\rangle \unlhd \bar{G}$, contradicting the fact that $\bar{G}$ satisfies the strong condition on normal subgroups. It follows that $[\bar{a}, \bar{x}]=(\overline{a b})^{2}$. Consequently,

$$
1=\left[a, x^{2}\right]=[a, x]^{2}[a, x, x]=[a, x]^{2}\left[a^{2} b^{2}, x\right]=[a, x]^{4}[b, x]^{2}=[b, x]^{2},
$$

since $\exp G^{\prime} \leq 4$. We have thus proved that $[b, x]^{2}=1$ for any $x \in G$, which completes the proof.

Example. The condition that the index of the centre is not a square cannot be dropped from the hypotheses in the previous theorem. Indeed, there are examples of $p$-groups of class 3 and arbitrarily high order satisfying the weak condition on normal subgroups for which $\left|Z_{2}(G): Z(G)\right|=p^{2}$. It suffices, for odd $p$, to consider 
the group $G_{n}=\left\langle a, b \mid a^{p^{3}}=b^{p^{n}}=1, a^{b}=a^{1+p}\right\rangle$ and, for $p=2, G_{n}=\langle a, b, c|$ $\left.a^{8}=b^{2^{n-1}}=c^{2}=1, a^{b}=a^{5}, a^{c}=a^{-1}, b^{c}=b\right\rangle$, where $n \geq 2$ in both cases.

\section{Proof of Theorem F}

Our aim now is to prove Theorem F. To this end, the structural information about the $p$-groups with the weak condition on normal subgroups and class 3 obtained in the previous section will be of fundamental importance.

Since Theorem F deals with several different cases, we split its proof into a number of results. We begin by studying groups with the strong or weak condition on normal subgroups with maximum nilpotency class. Recall that we have already proved in Proposition 4.1 that this maximum class is 3 or 4 , respectively.

Proposition 6.1. Let $G$ be a p-group.

(i) If $G$ satisfies the strong condition on normal subgroups and $c(G)=3$, then $|G: Z(G)|=p^{3}$.

(ii) If $G$ satisfies the weak condition on normal subgroups and $c(G)=4$, then $|G: Z(G)|=p^{4}$.

Proof. (i) Since $G^{\prime}$ is contained in any subgroup $N$ such that $Z(G)<N \leq Z_{2}(G)$ and $G^{\prime} \not Z Z(G)$, we derive that $G^{\prime} Z(G) / Z(G)$ has order $p$ and is in fact the only subgroup of order $p$ of $Z_{2}(G) / Z(G)$. It follows that $Z_{2}(G) / Z(G)$ is cyclic, and Theorem 5.2 yields that $\left|Z_{2}(G): Z(G)\right|=p$. On the other hand, the derived subgroup of $G / Z(G)$ has order $p$, and we obtain from Lemma 2.5 that $\left|G: Z_{2}(G)\right|=$ $p^{2}$. The result follows.

(ii) According to Lemma [5.1, $G / Z(G)$ satisfies the strong condition on normal subgroups. It then follows from (i) that $\left|G: Z_{2}(G)\right|=p^{3}$. On the other hand, if $\left|Z_{2}(G): Z(G)\right| \geq p^{2}$, then $G^{\prime} \leq Z_{2}(G)$ and $G$ has class $\leq 3$, a contradiction. Hence $\left|Z_{2}(G): Z(G)\right|=p$ and $|G: Z(G)|=p^{4}$.

Again, the $p$-groups of maximal class of order $p^{4}$ and $p^{5}$ show that the bounds for $|G: Z(G)|$ in the previous proposition cannot be improved. The following straightforward corollary will be useful.

Corollary 6.2. Let $G$ be a p-group that satisfies the strong condition on normal subgroups. If $|G: Z(G)|$ is a square, then $G$ has class $\leq 2$.

In order to prove Theorem $\mathrm{F}$, it only remains to bound $|G: Z(G)|$ when $G$ is a $p$-group of class 3 satisfying the weak condition on normal subgroups. We first need a couple of lemmas.

Lemma 6.3. Let $G$ be a p-group that satisfies the strong condition on normal subgroups. If $|G: Z(G)|$ is a square, then $Z(G / N)=Z(G) / N$ for any normal subgroup $N$ of $G$ not containing $G^{\prime}$.

Proof. First of all, we may assume that $G$ has class 2 by Corollary 6.2. Let $T$ be any normal subgroup of $G$. Since $[x, G] \leq T$ if and only if $[x, G] \leq T \cap G^{\prime}$, we deduce that $Z(G / T)$ and $Z\left(G /\left(T \cap G^{\prime}\right)\right)$ have the same inverse image in $G$. Thus it suffices to prove the property when $T$ is properly contained in $G^{\prime}$. Clearly, we may also assume that $T$ is maximal in $G^{\prime}$. If we choose $N$ to be a normal subgroup of $G$ maximal with respect to $N \cap G^{\prime}=T$, then again $Z(G / N)$ and $Z(G / T)$ have the same inverse image in $G$. So we only need to prove the lemma for this $N$. 
Let $K / N=Z(G / N)$. Then Lemma 4.2 yields that $K / N$ is cyclic. Since $G$ satisfies the strong condition on normal subgroups, we have that $N \leq Z(G)$ and $K / Z(G)$ is also cyclic. We know from Theorem D that $\exp G / Z(G)=p$, and consequently $|K / Z(G)| \leq p$. But $|G: Z(G)|$ is a square by hypothesis and $|G: K|$ is also a square by Lemma 4.2. Hence $K=Z(G)$, as we wanted to prove.

The following lemma generalizes Lemma 2.5 since any $p$-group $P$ whose derived subgroup has order $p$ necessarily satisfies the strong condition on normal subgroups: indeed, if $N \unlhd P$ and $P^{\prime} \not \leq N$, then $P^{\prime} \cap N=1$, and thus $N \leq Z(P)$.

Lemma 6.4. Let $P$ be a p-group that satisfies the strong condition on normal subgroups and such that $P / Z(P)$ has square order. If $\left|P^{\prime}\right|=p^{n}$ and $P$ is capable, then $|P: Z(P)| \leq p^{2 n}$.

Proof. The proof is partly inspired in the proof of Lemma 2.5. We may assume that $P$ has class 2 by Corollary 6.2. Since $P$ is capable, there is a group $G$ such that $P=G / Z(G)$. Let us use the bar notation in $P$. For $\bar{a} \in P \backslash Z(P)$, that is, $a \in G \backslash Z_{2}(G)$, let $C_{a}$ denote the inverse image in $G$ of $C_{P}(\bar{a})$.

Suppose first that $C_{P}(\bar{a})^{\prime}=P^{\prime}$ for all $a \in G \backslash Z_{2}(G)$. From the definition of $C_{a}$ we have that $\left[\langle a\rangle G^{\prime}, C_{a}\right] \leq Z(G)$. Consequently $\left[\langle a\rangle G^{\prime}, C_{a}, C_{a}\right]=1$, and it follows from P. Hall's three subgroup lemma that $C_{a}^{\prime}$ commutes with $a$. Since $C_{a}^{\prime} Z(G)=G^{\prime} Z(G)$, also $G^{\prime}$ commutes with $a$. But $a$ is any element in $G \backslash Z_{2}(G)$, so we conclude that $G^{\prime} \leq Z(G)$, a contradiction.

So we may assume that $C_{P}(\bar{a})^{\prime}$ is a proper subgroup of $P^{\prime}$ for some $a$. We can then embed $C_{P}(\bar{a})^{\prime}$ in a maximal subgroup $N$ of $P^{\prime}$. Put $K=P / N$ and let $D$ denote the image of $C_{P}(\bar{a})$ in this quotient. Since $|K: D|=\left|P: C_{P}(\bar{a})\right| \leq\left|P^{\prime}\right|=p^{n}$, we can find $x_{1}, \ldots, x_{n} \in K$ such that $K=\left\langle x_{1}, \ldots, x_{n}, D\right\rangle$. Now $D$ is abelian, and consequently the subgroup $E=D \cap\left(\bigcap_{i=1}^{n} C_{K}\left(x_{i}\right)\right)$ is central in $K$. It follows that

$$
|K: Z(K)| \leq|K: E| \leq|K: D| \prod_{i=1}^{n}\left|K: C_{K}\left(x_{i}\right)\right| \leq p^{2 n},
$$

because $\left|K: C_{K}\left(x_{i}\right)\right| \leq\left|K^{\prime}\right|=p$ for all $i$. Finally, since $P$ satisfies the strong condition on normal subgroups and $|P / Z(P)|$ is a square, Lemma 6.3 shows that $Z(K)=Z(P / N)=Z(P) / N$. It then follows that $|P: Z(P)|=|K: Z(K)| \leq p^{2 n}$, as desired.

The condition that $P$ satisfies the strong condition on normal subgroups is essential in the previous lemma, since Heineken [8] has constructed for any $n \geq 2$ a

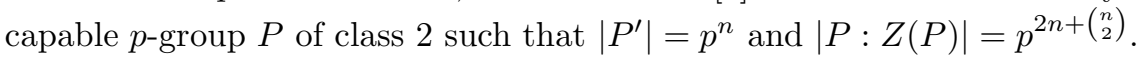

Proposition 6.5. Let $G$ be a p-group of class 3 satisfying the weak condition on normal subgroups. Then the following statements hold:

(i) If $\left|Z_{2}(G): Z(G)\right|=p$, then $|G: Z(G)|=p^{3}$.

(ii) If $\left|Z_{2}(G): Z(G)\right|=p^{2}$, then $|G: Z(G)|=p^{4}$ or $p^{6}$, according to whether $\left|G^{\prime} Z(G): Z(G)\right|=p$ or $p^{2}$.

Proof. Set $P=G / Z(G)$. We know from Theorem 5.2 that $Z(P) \cong C_{p}$ or $C_{p} \times$ $C_{p}$. Also, recall from Lemma 5.1 that $P$ satisfies the strong condition on normal subgroups.

If $|Z(P)|=p$ then $\left|P^{\prime}\right|=p$, and Lemma 2.5 shows that $|P: Z(P)|=p^{2}$. Hence $|G: Z(G)|=|P|=p^{3}$ in this case. Suppose then that $|Z(P)|=p^{2}$. This yields, 
according to Theorem [5.3, that $|P|$ is a square or, what is the same in this case, that $|P: Z(P)|$ is a square. If $\left|P^{\prime}\right|=p$ then $|P: Z(P)|=p^{2}$ by Lemma [2.5] and therefore $|G: Z(G)|=p^{4}$. Otherwise $\left|P^{\prime}\right|=p^{2}$, and we deduce that $|P: Z(P)|=p^{2}$ or $p^{4}$, by applying Lemma 6.4 But we cannot have $|P: Z(P)|=p^{2}$, since then $\left|P^{\prime}\right|=p$. Consequently $|P: Z(P)|=p^{4}$ and $|G: Z(G)|=p^{6}$ in this case.

Examples. (i) The groups given after Theorem 5.3 provide examples of groups of class 3 satisfying the weak condition on normal subgroups for which $|G: Z(G)|=p^{4}$.

(ii) Let $p$ be an odd prime, and consider the group $G=\left\langle a_{1}, \ldots, a_{7}\right\rangle$, where the generators have order $p$ and satisfy the following non-trivial commutator relations:

$$
\left[a_{3}, a_{1}\right]=a_{5}, \quad\left[a_{4}, a_{1}\right]=a_{6}, \quad\left[a_{5}, a_{1}\right]=a_{7}
$$

and

$$
\left[a_{3}, a_{2}\right]=a_{6}, \quad\left[a_{4}, a_{2}\right]=a_{5}^{i}, \quad\left[a_{6}, a_{2}\right]=a_{7}^{i},
$$

with $i$ a non-quadratic residue modulo $p$. Then $G$ has class 3 and satisfies the weak condition on normal subgroups, whereas $Z(G)=\left\langle a_{7}\right\rangle$ has index $p^{6}$ in $G$.

Next we see that, unlike for the odd primes, there are no 2-groups of class 3 satisfying the weak condition and such that the index of the centre equals $2^{6}$. This completes the proof of Theorem F.

Theorem 6.6. Let $G$ be a 2-group of class 3 satisfying the weak condition on normal subgroups. Then $|G: Z(G)|=2^{3}$ or $2^{4}$.

Proof. Assume $G$ is a counterexample to the theorem, and let $Q=G / Z(G)$. For ease of notation, we will write the elements of $Q$ without bars. Then Proposition 6.5 yields that $|Q|=2^{6}$ and that $Z(Q)=Q^{\prime}$ has order 4 . Thus $Z_{2}(G)=G^{\prime} Z(G)$. On the other hand, $Q$ has the strong condition on normal subgroups according to Lemma 5.1 Hence we may apply Lemma 6.3 and Theorem A to obtain that $[x, Q]=Q^{\prime}$ for any $x \in Q \backslash Z(Q)$.

After these preliminary considerations, we split the proof into a number of steps. Step 1. $\exp \left(G^{\prime} \cap Z(G)\right)=2$.

From Theorem 5.2 we have that $L=Z_{2}(G)$. Thus $[L, G]=\gamma_{3}(G)$, and $G / \gamma_{3}(G)$ has the strong condition on normal subgroups by Lemma 5.1 It follows from Theorem D that $\exp G^{\prime} / \gamma_{3}(G)=2$.

On the other hand, if we choose elements $u, v \in G$ such that $Z_{2}(G)=\langle u, v\rangle Z(G)$, then $N=\langle u, v\rangle \gamma_{3}(G)$ is a normal subgroup of $G$. Since $G$ has the weak condition, we derive that $G^{\prime} \leq N$ and $d\left(G^{\prime} / \gamma_{3}(G)\right) \leq 2$. Hence $\left|G^{\prime}: \gamma_{3}(G)\right| \leq 4$. But $\gamma_{3}(G) \leq G^{\prime} \cap Z(G)$ and $\left|G^{\prime}: G^{\prime} \cap Z(G)\right|=\left|G^{\prime} Z(G): Z(G)\right|=4$; therefore $G^{\prime} \cap Z(G)=\gamma_{3}(G)$. We conclude from Lemma 5.1 that $\exp \left(G^{\prime} \cap Z(G)\right)=2$.

Step 2. $Q$ has five maximal abelian subgroups, all of which have order $2^{4}$. Besides, the product of any two distinct ones of them is the whole of $Q$.

For any $x \in Q \backslash Z(Q)$ we have seen that $[x, Q]=Q^{\prime}$, and consequently $C_{Q}(x)$ has order $2^{4}$. Then $C_{Q}(x)=\langle x, y\rangle Z(Q)$ for some $y$, and this subgroup is abelian. In fact, it is obviously a maximal abelian subgroup of $Q$. Conversely, suppose $A$ is a maximal abelian subgroup of $Q$. If we choose $x \in A \backslash Z(Q)$, then clearly $A=C_{Q}(x)$. Thus we have proved that the maximal abelian subgroups of $Q$ are the centralizers of the non-central elements. Now an easy counting argument shows that $Q$ has five different maximal abelian subgroups. Finally, if $A$ and $B$ are two different maximal abelian subgroups of $Q$, then they cannot have a common non-central element. Consequently $A \cap B=Z(Q)$, and it is immediate that $Q=A B$. 
Step 3. If $A$ and $B$ are two different maximal abelian subgroups of $Q$, then $\mho_{1}(A) \cap$ $\mho_{1}(B)=1$.

Write $A=X / Z(G), B=Y / Z(G)$ and $\mho_{1}(A) \cap \mho_{1}(B)=T / Z(G)$. We have to see that $T=Z(G)$.

Since $A$ is abelian, we have that $X^{\prime} \leq Z(G)$, and $X$ has class $\leq 2$. Then we derive from Lemma 2.2 that $\left[\mho_{1}(X), X\right]=\mho_{1}\left(X^{\prime}\right)=1$, since $X^{\prime} \leq G^{\prime} \cap Z(G)$ has exponent 2 by Step 1 . Now by the definition of $T$ we have that $T \leq \mho_{1}(X) Z(G)$, and consequently $[T, X]=1$. Similarly $[T, Y]=1$. Finally, since $A$ and $B$ are different, we deduce from the previous step that $Q=A B$, that is, $G=X Y$. Thus $T \leq Z(G)$.

Step 4. Final contradiction.

Since $Q$ satisfies the strong condition on normal subgroups, we know from Theorem D that $\exp Q / Z(Q)=2$, and consequently $\mho_{1}(Q) \leq Z(Q) \cong C_{2} \times C_{2}$. So if $A$ is any of the maximal abelian subgroups of $Q, \mho_{1}(A)$ has to be one of the five subgroups of $Z(Q)$. But we know from Step 2 that $Q$ has five maximal abelian subgroups, so the only way to avoid a contradiction with Step 3 is if there exist two different maximal abelian subgroups $A$ and $B$ of $Q$ such that $\mho_{1}(A)=\mho_{1}(B)=1$.

Write $A=\langle x, y\rangle Z(Q)$. Since $Q=A B$, we have that $Q^{\prime}=[x, Q]=[x, B]$ and also $Q^{\prime}=[y, B]$. Thus we may choose $z, t \in B$ such that $[x, z]=[y, t] \neq 1$. Let $C=C_{Q}(x z)$ and $D=C_{Q}(y t)$, which are maximal abelian subgroups of $Q$. Observe that

$$
(x z)^{2}=x^{2} z^{2}[z, x]=[z, x] \quad \text { and } \quad(y t)^{2}=y^{2} t^{2}[t, y]=[t, y]
$$

are equal, so according to the previous step we have that $C=D$. But then $x z$ and $y t$ commute, and therefore $1=[x z, y t]=[x, t][z, y]$. Consequently $[x y, z t]=$ $[x, z][x, t][y, z][y, t]=1$, which is a contradiction, since $C_{Q}(x y)=A$.

We have remarked in the introduction that the proofs we provide for Theorems $\mathrm{D}$ to $\mathrm{G}$ on groups with either of the conditions on normal subgroups are characterfree. This seems not to be true for Theorem 6.6, since at the beginning of its proof we appeal to Theorem A. Nevertheless, we only need that (v) implies (iii) in that theorem, which is completely straightforward and does not require the character theoretical part (i) at all.

\section{Proof of Theorem G}

In this section we prove Theorem $\mathrm{G}$, that is, that the order of a group $G$ with the strong or weak condition on normal subgroups and class greater than 2 is bounded unless $G$ has the weak condition and $|G: Z(G)|=p^{3}$ or $p^{4}$. Observe that Theorem $\mathrm{G}$ cannot be extended to these two cases. On the one hand, given any $p$-group $P$ of maximal class and order $p^{4}$ and any abelian $p$-group $A$, the group $G=P \times A$ has class 3, satisfies the weak condition and $|G: Z(G)|=p^{3}$. On the other hand, the examples after Theorem 5.3 show that the order can be arbitrarily high when $|G: Z(G)|=p^{4}$.

We split the proof of Theorem $G$ into the two theorems below. The first of them considers the case where the class of $G$ is maximum with respect to the condition it satisfies, that is, $c(G)=3$ if $G$ satisfies the strong condition and $c(G)=4$ if it satisfies the weak condition. The possibility of getting a bound for $|G|$ in this case was pointed out to us by A. Mann. The second theorem deals with the remaining case, where $G$ satisfies the weak condition, has class 3 , and $|G: Z(G)|=p^{6}$. 
We already know that the $p$-groups of maximal class and order $p^{4}$ and $p^{5}$ are examples of groups with the strong and weak condition, respectively, for which the nilpotency class is as large as possible. Note also that Proposition 6.1 tells us that for a group $G$ of that kind $G / Z(G)$ has maximal class. Our next result then shows that $G$ itself is almost of maximal class. In fact, if $p=2$, then $G$ necessarily has maximal class.

Theorem 7.1. Let $G$ be a p-group.

(i) If $G$ satisfies the strong condition on normal subgroups and $c(G)=3$, then $|G| \leq p^{5}$. Furthermore, if $p=2$ then $|G|=2^{4}$, that is, $G$ has maximal class.

(ii) If $G$ satisfies the weak condition on normal subgroups and $c(G)=4$, then $|G| \leq p^{6}$. Furthermore, if $p=2$ then $|G|=2^{5}$, that is, $G$ has maximal class.

Proof. Recall that, as explained before the theorem, $G / Z(G)$ is a $p$-group of maximal class in any case.

(i) Since $\left|Z_{2}(G): Z(G)\right|=p$, we may write $Z_{2}(G)=\langle x, Z(G)\rangle$ for some $x$ such that $x^{p} \in Z(G)$. Then $N=\langle x\rangle[x, G]$ is normal in $G$, and by hypothesis $G^{\prime} \leq N$. It follows that

$$
G^{\prime}=G^{\prime} \cap N=\left(G^{\prime} \cap\langle x\rangle\right)[x, G],
$$

by Dedekind's Law. But $x^{p}$ and $[x, G]$ are central while $G^{\prime}$ is not; therefore $G^{\prime} \cap\langle x\rangle=$ $\langle x\rangle$, that is, $x \in G^{\prime}$. Since $x$ can be any element of $Z_{2}(G) \backslash Z(G)$, we get that $G^{\prime}=Z_{2}(G)$.

Hence we have that $\left|G: G^{\prime}\right|=p^{2}$. If $p=2$ this implies that $G$ has maximal class (see [10, Theorem III.11.9]) and $|G|=2^{4}$. In general, if $G=\left\langle a, b, G^{\prime}\right\rangle$ then by [10] Lemma III.1.11] we have that $G^{\prime}=\left\langle[a, b], \gamma_{3}(G)\right\rangle$ and $\gamma_{3}(G)=\langle[a, b, a],[a, b, b]\rangle$. Consequently $\left|G^{\prime}: \gamma_{3}(G)\right|=p$ and $\left|\gamma_{3}(G)\right| \leq p^{2}$, which proves that $|G| \leq p^{5}$.

(ii) Choose $x \in G$ such that $Z_{3}(G)=\left\langle x, Z_{2}(G)\right\rangle$. Let $T$ be the normal closure of $[x, G]$ in $G$ and $N=\langle x\rangle T$, which is normal in $G$. Since $G / Z(G)$ has maximal class, we have that $[x, G] Z(G)=Z_{2}(G)$, and consequently $N Z(G)=Z_{3}(G)$. Thus $|N Z(G): Z(G)|=p^{2}$, and by hypothesis $G^{\prime} \leq N$. Since $T$ is contained in both $G^{\prime}$ and $Z_{2}(G)$, we can argue as in part (i) to derive that $x \in G^{\prime}$. Hence $G^{\prime}=Z_{3}(G)$ and $\left|G: G^{\prime}\right|=p^{2}$.

If $p=2$ we deduce again that $G$ has maximal class. In the general case, we have as in the proof of (i) that $\left|G^{\prime}: \gamma_{3}(G)\right|=p$ and $\left|\gamma_{3}(G): \gamma_{4}(G)\right| \leq p^{2}$. Since $G / Z(G)$ is a group of maximal class of order $p^{4}$, it has an abelian maximal subgroup (consider the centralizer of its second centre). Consequently, $G$ has a maximal subgroup $M$ such that $M^{\prime} \leq Z(G)$ and, in particular, $\left[M, G^{\prime}, G\right]=1$. On the other hand, $\left[G, M, G^{\prime}\right] \leq\left[G^{\prime}, G^{\prime}\right]=\left[G^{\prime}, \gamma_{3}(G)\right] \leq \gamma_{5}(G)=1$, where the equality follows from $G^{\prime} / \gamma_{3}(G)$ being cyclic. Now the three subgroup lemma yields that $\left[G^{\prime}, G, M\right]=1$, that is, $\left[\gamma_{3}(G), M\right]=1$. By taking into account again that $G / Z(G)$ has maximal class, we derive that $\gamma_{3}(G / Z(G))=\gamma_{3}(G) Z(G) / Z(G)$ has order $p$, and hence $\left|G: \gamma_{3}(G) Z(G)\right|=p^{3}$. Since $\left|G: \gamma_{3}(G)\right|=p^{3}$, it follows that $Z(G) \leq$ $\gamma_{3}(G)$ and $\left|\gamma_{3}(G): Z(G)\right|=p$. Thus we may write $\gamma_{3}(G)=\langle y, Z(G)\rangle$, and also $G=\langle a, M\rangle$, since $M$ is maximal in $G$. As we have proved that $\gamma_{3}(G)$ and $M$ commute elementwise, we deduce that $\gamma_{4}(G)=\langle[y, a]\rangle$ has order $p$. We conclude that $|G| \leq p^{6}$.

As is well-known, a 2-group of maximal class is a dihedral, semidihedral or generalized quaternion group. Thus these are the only 2-groups that appear in the previous theorem. Next we see that the bounds obtained for $p>2$ are sharp. 
Examples. (i) Let $p>2$ be a prime and let $\mathbb{Z}_{p}$ denote the ring of $p$-adic integers. Consider the subgroup $G$ of $S L_{2}\left(\mathbb{Z}_{p}\right)$ formed by the matrices $\left(\begin{array}{ll}a & b \\ c & d\end{array}\right)$ such that $a \equiv$ $d \equiv 1(\bmod p)$ and $c \equiv 0(\bmod p)$. In other words, $G$ is the inverse image, under the natural homomorphism from $S L_{2}\left(\mathbb{Z}_{p}\right)$ onto $S L_{2}\left(\mathbb{F}_{p}\right)$, of the Sylow $p$-subgroup of $S L_{2}\left(\mathbb{F}_{p}\right)$ formed by the upper unitriangular matrices. This group is called the binary p-adic group and is studied in detail in [10, Chapter III.17]. We only need to use here that $a_{i}=\left|\gamma_{i}(G): \gamma_{i+1}(G)\right|$ is finite for all $i$ and that the sequence $\left\{a_{i}\right\}_{i \geq 1}$ is periodic with the following pattern: $p^{2}, p, p^{2}, p, p^{2}, p, \ldots$ It is easy to deduce from this fact that the upper and lower central series of $K=G / \gamma_{4}(G)$ coincide. This in turn implies that the group $K$, which has class 3 and order $p^{5}$, satisfies the strong condition on normal subgroups. Alternatively, one can take for $p>3$ the free nilpotent group of class 3 and exponent $p$ in two generators.

(ii) Let $p>3$ be a prime and consider a vector space $L=\left\langle a_{1}, \ldots, a_{6}\right\rangle$ of dimension 6 over $\mathbb{F}_{p}$. Let us define a multiplication [, ] on the basis elements for which the non-trivial products are given by

$$
\left[a_{1}, a_{2}\right]=a_{3}, \quad\left[a_{1}, a_{3}\right]=a_{4}, \quad\left[a_{2}, a_{3}\right]=a_{5}, \quad\left[a_{1}, a_{4}\right]=a_{6} .
$$

Then [,] extends by bilinearity to an alternating product in $L$, and it is easy to check that $(L,[]$,$) is in fact a Lie algebra. Furthermore, L^{\prime}=\left\langle a_{3}, \ldots, a_{6}\right\rangle$, $L^{3}=\left\langle a_{4}, a_{5}, a_{6}\right\rangle, L^{4}=\left\langle a_{6}\right\rangle$ and $L^{5}=0$, so that $L$ is nilpotent of class 4 . Also, $Z(L)=\left\langle a_{5}, a_{6}\right\rangle$. Then we may apply Lazard's correspondence, which enables us to transform the underlying set of $L$ in a $p$-group $G$ thanks to the Baker-Hausdorff formula. (For a detailed account of both the Baker-Hausdorff formula and Lazard's correspondence, see [16].) Then $G$ has order $p^{6}$ and class 4 , and $\left|G: G^{\prime}\right|=\mid \gamma_{3}(G)$ : $\gamma_{4}(G)\left|=p^{2},\right| G^{\prime}: \gamma_{3}(G)|=| \gamma_{4}(G) \mid=p$. On the other hand, $Z(G)$ is contained in $\gamma_{3}(G)$ and has order $p^{2}$. It is not difficult to derive from all this information that $G$ has the weak condition on normal subgroups. A similar construction works to give an example for $p>3$ in the case of the strong condition, as in (i): it suffices to apply Lazard's correspondence to the Lie algebra $L / L^{4}$. Nevertheless, the group we obtain in this way is again the free nilpotent group of class 3 and exponent $p$ in two generators.

(iii) A search in the database of groups of order $3^{6}$ available in the algebra system GAP [19] shows that there are groups among them of class 4 and satisfying the weak condition on normal subgroups.

In the second theorem we have contented ourselves with showing that the order of the group is bounded; we have not tried to obtain a sharp bound.

Theorem 7.2. Let $G$ be a p-group of class 3 satisfying the weak condition on normal subgroups. If $|G: Z(G)|=p^{6}$, then $G$ has bounded order. In fact, $|G| \leq p^{18}$.

Proof. First of all, observe from Proposition 6.5 that $\left|Z_{2}(G): Z(G)\right|=p^{2}$ and $Z_{2}(G)=G^{\prime} Z(G)$ is abelian. We are going to prove that $G^{\prime}=Z_{2}(G)$, whence $\left|G: G^{\prime}\right|=p^{4}$. Then it follows from [10, Lemma III.1.11] that $\left|G^{\prime}: \gamma_{3}(G)\right| \leq p^{6}$ and, since $\left|G^{\prime}: Z(G)\right|=p^{2}$, also that $\left|\gamma_{3}(G): \gamma_{4}(G)\right| \leq p^{8}$. But $G$ has class 3, so we conclude that $|G| \leq p^{18}$.

In order to see that $G^{\prime}=Z_{2}(G)$, it suffices to prove that any $x \in Z_{2}(G) \backslash Z(G)$ belongs to $G^{\prime}$. To this end, choose an element $y$ such that $Z_{2}(G)=\langle x, y\rangle Z(G)$ and consider the subgroup $H=\langle x, y\rangle$. We have that $\Phi(H)=\left\langle x^{p}, y^{p}\right\rangle \leq H \cap Z(G)$. Since $|H: H \cap Z(G)|=|H Z(G): Z(G)|=p^{2}$ and $d(H)=2$, we deduce that $\Phi(H)=H \cap Z(G)$. 
Now $N=H[H, G]$ is a normal subgroup of $G$ such that $|N Z(G): Z(G)|=p^{2}$. Since $G$ satisfies the weak condition on normal subgroups, we derive that $G^{\prime} \leq N$ and $G^{\prime}=G^{\prime} \cap N=\left(G^{\prime} \cap H\right)[H, G]$ by Dedekind's Law. Consequently $H Z(G)=$ $G^{\prime} Z(G)=\left(G^{\prime} \cap H\right) Z(G)$. It follows that $H=\left(G^{\prime} \cap H\right)(Z(G) \cap H)=\left(G^{\prime} \cap H\right) \Phi(H)$ and therefore $H=G^{\prime} \cap H$. In particular $x \in G^{\prime}$, as desired.

\section{Proof of Theorems B And C}

We are now ready to return to our character theoretical problem and prove Theorems B and C, which will follow easily by using the results in the previous sections. Note that the statements in part (ii) of these two theorems correspond to $G$ satisfying the strong or weak condition on normal subgroups, respectively.

In the next proposition we prove that (ii) implies (i) in Theorem B. Recall that the fact that (ii) follows from (i) was proved in the introduction.

Proposition 8.1. Let $G$ be a p-group such that $|G: Z(G)|=p^{2 n}$ is a square. If $G$ satisfies the strong condition on normal subgroups, then $\operatorname{cd}(G)=\left\{1, p^{n}\right\}$.

Proof. It suffices to combine Lemma 6.3 with Theorem A.

We want to remark that the previous simple proof eventually depends on Theorem $\mathrm{D}$ and part of Theorem $\mathrm{F}$, which were necessary to prove Lemma 6.3

Example. It seems natural to ask whether the strong condition on normal subgroups in a $p$-group $G$ of class 2 already implies that $|G: Z(G)|$ is a square. This is not the case, as the following examples show. For odd $p$, choose a non-quadratic residue $i$ modulo $p$ and consider the group

$$
G=\left\langle a, b, c \mid a^{p^{2}}=b^{p^{2}}=c^{p}=1,[a, b]=1,[a, c]=b^{p},[b, c]=a^{i p}\right\rangle,
$$

and, for $p=2$, take

$$
G=\left\langle a, b, c \mid a^{4}=b^{4}=c^{2}=1,[a, b]=1,[a, c]=b^{2},[b, c]=(a b)^{2}\right\rangle .
$$

(Note that in both cases the index of $Z(G)=\left\langle a^{p}, b^{p}\right\rangle$ is $p^{3}$.)

One could perharps guess that, if the index of the centre of a $p$-group of class 2 satisfying the strong condition on normal subgroups is not a square, then that index is bounded. We may see that this is not the case with the help of the following proposition.

Proposition 8.2. Let $H$ and $K$ be two p-groups of class 2 such that

(i) $H$ satisfies the strong condition on normal subgroups,

(ii) $K$ is semiextraspecial, and

(iii) $H^{\prime}$ and $K^{\prime}$ are isomorphic.

Then a central product of $H$ and $K$ with amalgamated derived subgroups also satisfies the strong condition.

Proof. Let $G$ be a central product of $H$ and $K$ with amalgamated derived subgroups. Suppose that $N$ is a normal non-central subgroup of $G$, and let us see that $G^{\prime} \leq N$. Choose $x=h k \in N \backslash Z(G)$, where $h \in H$ and $k \in K$.

If $k \notin Z(K)$ then $[k, K]=K^{\prime}$, since $K$ is semiextraspecial. But $[k, K]=[x, K] \leq$ $N$ and $K^{\prime}=G^{\prime}$, whence $G^{\prime} \leq N$ follows in this case. So we may assume that $k \in Z(K)$ and $h \notin Z(H)$. Since $H$ satisfies the strong condition, we obtain that $H^{\prime} \leq\langle h\rangle[h, H]$ and $H^{\prime}=\left(\langle h\rangle \cap H^{\prime}\right)[h, H] \leq\left\langle h^{p}\right\rangle[h, H]$. But $K$ is semiextraspecial, 
so we know that $\exp Z(K)=\exp K^{\prime}=p$ and consequently $h^{p}=h^{p} k^{p}=x^{p} \in N$. On the other hand, $[h, H]=[x, H] \leq N$, and thus $G^{\prime}=H^{\prime} \leq N$ also in this case.

The previous proposition does not hold if we simply suppose in (ii) that $K$ has the strong condition on normal subgroups. Indeed, the central product of one of the groups in the example above with itself, with the derived subgroup amalgamated via the identity map, does not fulfill the strong condition.

If we now consider a semiextraspecial group $K$ such that $\left|K^{\prime}\right|=p^{2}$ (for example, a Sylow $p$-subgroup of $S L\left(3, p^{2}\right)$, see [3, Lemma 4]), we may perform a central product of the corresponding group in the example above with any number of copies of $K$, amalgamating the derived subgroups. According to Proposition 8.2 the resultant group satisfies the strong condition, and it is clear that the index of the centre will be an arbitrarily large non-square.

In 20, Proposition 1.3] Verardi proved that semiextraspecial $p$-groups have the strong condition on normal subgroups and, as a partial converse, that any nonabelian special group of exponent $p$ satisfying the strong condition is necessarily semiextraspecial. This can be viewed as a very particular case of Theorem B. In fact, if we take into account the result of Noritzsch cited in the introduction, it is obvious that Theorem B yields the following characterization of semiextraspecial $p$-groups in terms of their normal subgroups.

Corollary 8.3. Let $G$ be a p-group. Then the following conditions are equivalent:

(i) $G$ is semiextraspecial (i.e. a Camina p-group of class 2 ).

(ii) $|G: Z(G)|$ is a square, $G^{\prime}=Z(G)$, and $G$ satisfies the strong condition on normal subgroups.

Note however that, unlike Verardi, we need to impose the condition that $|G: Z(G)|$ is a square to obtain that $G$ is semiextraspecial: consider otherwise the same examples above.

We may also apply Lemma 6.4 to semiextraspecial groups and generalize the well-known fact that a capable extraspecial $p$-group has order $p^{3}$.

Corollary 8.4. Any capable semiextraspecial p-group with derived subgroup of or$\operatorname{der} p^{n}$ has order $p^{3 n}$.

Proof. We know that a semiextraspecial group $G$ satisfies the strong condition on normal subgroups, and that $|G: Z(G)|$ is a square. If $\left|G^{\prime}\right|=p^{n}$, then Lemma 6.4 yields that $\left|G: G^{\prime}\right|=|G: Z(G)| \leq p^{2 n}$. But, as Beisiegel proved in [3, Theorem 1], the reverse inequality holds in any semiextraspecial group with derived subgroup of order $p^{n}$. We conclude that $\left|G: G^{\prime}\right|=p^{2 n}$ and $|G|=p^{3 n}$.

Our last objective is to confirm that Theorem $\mathrm{C}$ holds. We begin by proving the straightforward part of it: that (ii) is a consequence of (i).

Proposition 8.5. Let $G$ be a p-group such that $|G: Z(G)|=p^{2 n+1}$ is not a square. If $\operatorname{cd}(G)=\left\{1, p^{n}\right\}$, then $c(G)=2$ or 3 , and $G$ satisfies the weak condition on normal subgroups.

Proof. Since $G / Z_{2}(G)$ is a $p$-group of order $\leq p^{2 n}$ and $\operatorname{cd}\left(G / Z_{2}(G)\right) \subseteq\left\{1, p^{n}\right\}$, we deduce that $G / Z_{2}(G)$ is abelian and $c(G) \leq 3$. 
Now let $N$ be any normal subgroup of $G$ such that $G^{\prime} \not \leq N$. Then $\operatorname{cd}(G / N)=$ $\left\{1, p^{n}\right\}$, and consequently

$$
\begin{aligned}
p^{2 n} & \leq|G / N: Z(G / N)| \leq|G / N: N Z(G) / N| \\
& =|G: N Z(G)| \leq|G: Z(G)|=p^{2 n+1} .
\end{aligned}
$$

It follows that $|N Z(G): Z(G)| \leq p$, and $G$ satisfies the weak condition on normal subgroups.

The following proposition concludes the proof of Theorem C.

Proposition 8.6. Let $G$ be a p-group such that $|G: Z(G)|=p^{2 n+1}$ is not a square. If $G$ satisfies the weak condition on normal subgroups, then $\operatorname{cd}(G)=\left\{1, p^{n}\right\}$.

Proof. First of all, observe that Theorem F implies that either $G$ has class 2 or it has class 3 and $|G: Z(G)|=p^{3}$. In the latter case it is clear that $\operatorname{cd}(G)=\{1, p\}$, so we may assume that $c(G)=2$. If $\chi \in \operatorname{Irr}(G)$ is non-linear, we know that $\chi(1)^{2}=|G: Z(\chi)|$. Let us prove that $|Z(\chi): Z(G)|=p$, whence $\chi(1)=p^{n}$ follows.

Put $K=\operatorname{Ker} \chi$. Since $G^{\prime} \not \leq K$, we have $|K Z(G): Z(G)| \leq p$. On the other hand, $Z(\chi) / K$ is cyclic, and so $Z(\chi) / K Z(G)$ is too. As we know from Theorem $\mathrm{D}$ that $\exp G / Z(G)=p$, we derive that $|Z(\chi): K Z(G)| \leq p$. Thus $|Z(\chi): Z(G)| \leq p^{2}$ and, taking into account that $|G: Z(\chi)|$ is a square and $|G: Z(G)|$ is not, we necessarily have that $|Z(\chi): Z(G)|=p$.

Now that Theorems $\mathrm{B}$ and $\mathrm{C}$ are proved, we can show that if $P$ is a $p$-group satisfying either the strong or weak condition on normal subgroups and $|P: Z(P)|$ is a square or is not, respectively, then $P \times A$ satisfies the same condition on normal subgroups for any abelian $p$-group $A$ : it suffices to note that the condition on the character degrees is preserved by that direct product.

\section{REFERENCES}

[1] W. Bannuscher, Über Gruppen mit wenigen irreduziblen Charakteren I, II, Math. Nachr. 153 (1991), 79-84, 131-135. MR 92i:20008

[2] W. BANNUSChER, Über Gruppen mit genau zwei irreduziblen Charaktergraden I, II, Math. Nachr. 154 (1991), 253-263. MR 92k:20012

[3] B. Beisiegel, Semi-extraspezielle p-Gruppen, Math. Z. 156 (1977), 247-254. MR 57:12683

[4] R. DARK, C.M. Scoppola, On Camina groups of prime power order, J. Algebra 181 (1996), 787-802. MR 97b:20022

[5] L. Di Martino, M.C. Tamburini, Some remarks on the degrees of faithful irreducible representations of a finite p-group, Geom. Dedicata 41 (1992), 155-164. MR 93c:20014

[6] M. Hall JR., J.K. Senior, "The Groups of Order $2^{n}(n \leq 6)$ ", MacMillan, New York, 1964. MR 29:5889

[7] P. Hall, The classification of prime-power groups, J. Reine Angew. Math. 182 (1940), 130141. MR 2:211b

[8] H. Heineken, Nilpotent groups of class 2 that can appear as central quotient groups, Rend. Sem. Mat. Univ. Padova 84 (1990), 241-248. MR 92c:20068

[9] R.B. Howlett, I.M. IsaAcs, On groups of central type, Math. Z. 179 (1982), 555-569. MR 83j:20020

[10] B. Huppert, "Endliche Gruppen I", Springer-Verlag, Berlin/Heildelberg/New York, 1967. MR 37:302

[11] B. Huppert, "Character Theory of Finite Groups", de Gruyter, Berlin/New York, 1998. MR 99j:20011

[12] I.M. IsAaCS, "Character Theory of Finite Groups", Dover, New York, 1994. MR 57:417 (1st ed.)

[13] I.M. IsAaCS, D.S. PASSman, A characterization of groups in terms of the degrees of their characters, Pacific J. Math 15 (1965), 877-903. MR 33:199 
[14] I.M. IsaACS, D.S. Passman, A characterization of groups in terms of the degrees of their characters II, Pacific J. Math 24 (1968), 467-510. MR 39:2864

[15] R. James, The groups of order $p^{6}$ (p an odd prime), Math. Comp. 34 (1980), 613-637. MR 84a:20024

[16] E.I. Khukhro, "p-Automorphisms of Finite $p$-Groups", London Math. Soc. Lecture Note Ser., vol. 246, Cambridge University Press, Cambridge, 1997. MR 99d:20029

[17] A. Mann, Minimal characters of p-groups, J. Group Theory 2 (1999), 225-250. MR 2000f:20007

[18] T. Noritzsch, Groups having three complex irreducible character degrees, J. Algebra $\mathbf{1 7 5}$ (1995), 767-798. MR 96d:20010

[19] M. SchÖNeRT ET AL., "GAP - Groups, Algorithms, and Programming", Lehrstuhl D für Mathematik, Rheinisch Westfälische Technische Hochschule, Aachen, fifth edition, 1995.

[20] L. Verardi, Gruppi semiextraspeciali di esponente p, Ann. Mat. Pura Appl. 148 (1987), 131-171. MR 89h:20033

Departamento de Matemáticas, Universidad del País Vasco, 48080 Bilbao (Spain)

E-mail address: mtpfealg@lg.ehu.es

Departamento de Matemáticas, Universidad del País Vasco, 48080 Bilbao (Spain)

E-mail address: mtbmoqua@lg.ehu.es 